\title{
An Anthropomimetic Approach to High Performance Traction Control
}

\author{
William Kirchner ${ }^{1}$ \\ Steve C. Southward \\ Graduate Research Assistant, Department \\ of Mechanical Engineering \\ 2 Associate Professor, Department of \\ Mechanical Engineering \\ Virginia Polytechnic Institute and State \\ University, Blacksburg, VA \\ Received 2011/02/09 \\ Accepted 2011/05/06

\section{Abstract} \\ The ability to learn and adapt to changing environmental conditions, as well as develop perceptive models based on \\ stimulus-response data, provides expert human drivers with significant advantages. When it comes to bandwidth, \\ accuracy, and repeatability, automatic control systems have clear advantages over humans; however, most high \\ performance control systems lack many of the unique abilities of a human expert. This paper documents our first \\ step toward the development of a novel automatic traction control algorithm using an anthropomimetic approach. \\ The primary objective of this approach was to synthesize a high performance longitudinal traction control system by \\ incorporating desirable human behavior distilled from human-in-the-loop (HIL) testing on a 6-DOF driving simulator. \\ The proposed control algorithm was developed in a general framework, and applied to the specific task of longitudinal \\ traction control. Simulation results confirm that the proposed anthropomimetic traction control algorithm provides \\ improved performance relative to a well-tuned conventional PID-based traction control algorithm. Results are also \\ compared with the HIL response data from a behavioral study. \\ Keywords \\ traction control $\cdot$ anthropomimetic $\cdot$ vehicle dynamics $\cdot$ human in the loop $\cdot$ adaptive control
}

\section{Introduction}

One functional definition for high performance traction control is a driver assist technology that enables ground vehicles to be driven from point $A$ to point $B$ in minimum time, barring engine power limitations. Achieving this objective requires maximization of the vector sum of the longitudinal and lateral traction forces at each tire-ground interface, also known as the tire patch. This can be a very challenging task for a human driver considering the significant variability in the terrain and the general lack of knowledge about the tire-ground interface. Even commercially available automatic traction control systems are designed with restrictive assumptions such as relative uniformity in the ground surface, which ultimately limits the operational envelope [1-7]

Motorsports applications have reaped the most value from automatic traction control largely because the tire-ground interface in those applications generally has less variability [8], and is subsequently relatively better understood through extensive testing [9]. Some form of automatic traction control system (TCS), or its more prevalent complement an anti-lock braking system (ABS), is also available on a wide range of passenger cars and trucks [5, 7]. Defense and civil applications of Unmanned Ground Vehicle (UGV) technology are rapidly progressing to the point where high-performance traction control systems for both acceleration and braking will be necessary to achieve future mobility and mission needs [10]. Existing UGV platforms are typically restricted to operate within a conservative performance envelope relative to traction at the tire-ground interface. Performance is commonly sacrificed in favor of stability as a mechanism for accommodating the interface uncertainties. Traction control solutions that offer higher levels of performance than what is currently available today will provide significant value for a wide range of ground vehicle applications.

Humans possess the unique ability to learn and adapt to uncertainty when performing tasks in a changing environment. With regard to ground vehicle traction, one need only look at professional drifting motorsports for examples of expert human drivers who have developed an ability to execute precise maneuvers while operating under conditions that non-expert drivers would consider dangerous or unstable. These and other expert human drivers are able to control their vehicles at high performance traction levels without direct sensing of speed, slip, or traction forces. Clearly human drivers rely on an information-rich set of indirect inputs such as visual, audible, inertial, and tactile cues, along with some form of internal representation of the dynamics and environmental conditions developed through reinforcement learning. The primary motivation for this research effort began with a fundamental belief that human experts do possess some valuable behavioral traits which can be exploited to improve traction control performance, even though humans generally do not possess the bandwidth, accuracy, or repeatability of an automatic control system. Borrowing nomenclature developed in the robotics field by Holland [11, 12], we propose to develop anthropomimetic solutions for high performance traction control. Literally translated as direct copying of desirable human behavior, this approach first requires the acquisition and distillation of pertinent human behavioral responses. The approach presented in this paper should be considered as a first step in the development of a truly anthropomimetic solution, where an adaptive control algorithm has been designed to be responsive to the same correlated input signals that a human expert would rely on, with the objective of maximizing traction. This work establishes a foundation for the ultimate challenge to synthesize anthropomimetic control algorithms that are capable of learning and adapting to an uncertain time varying environment. 


\section{HIL Traction Control Case Study}

The Cruden 6-DOF full-motion driving simulator located at VIPER Service [13], was utilized as a Human-In-the-Loop (HIL) test platform to develop a novel approach for synthesizing traction control algorithms designed to mimic the desirable behavior of human expert drivers. For this study, the driving simulator was used to measure human behavioral responses under controlled conditions. Recognizing that any simulation platform is by definition only an approximation of reality, the simulation platform used in this study has a high level of objective fidelity. Based on our experience and the feedback from expert drivers who train on this simulator, we believe that the perceptual fidelity is also relatively high, even though this is a very difficult metric to evaluate [30-33]. The VIPER driving simulator enables human drivers to experience the sight, sound, and tactile feedback at the steering wheel. Drivers also experience the pitch, roll, yaw, heave, surge, and sway motions associated with simulated vehicles in response to steering, braking, throttle, and gear selection commands provided by the driver as well as terrain inputs to the tires.
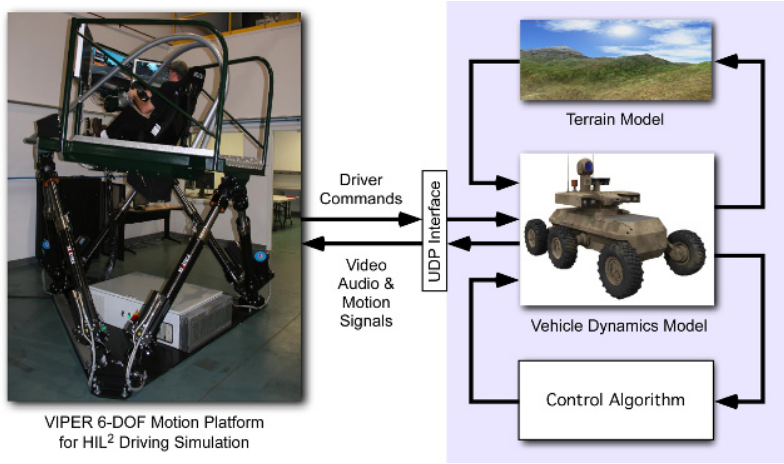

Figure 1. A HIL driving simulator platform was used to collect behavioral data from human expert drivers.

Figure 1 shows the basic simulation-mode operation where a human driver on the driving simulator (LHS of Figure 1) commands a virtual vehicle within a virtual terrain environment (RHS of Figure 1). Although not used in this particular study, the platform also allows for algorithms such as traction control to be integrated into the HIL simulation for prototype evaluation. A large selection of virtual vehicles was available for use in a variety of virtual environments, where each vehicle model is a full multi-body dynamic system including suspension kinematics, nonlinear tire dynamics, engine torque curves, and aero maps. The highest fidelity vehicle model available at VIPER was used for this study in order to maximize the realism experienced by the driver. Table 1 lists the physical performance specifications associated with the VIPER driving simulator. This table clearly indicates some of the limitations associated with the use of a simulation platform.

Since the primary purpose of this study was to establish the feasibility of an anthropomimetic approach to control system synthesis, a simple HIL driving task was designed with only one pertinent human command input in order to isolate one key aspect of traction control. This driving task was designed to study how human drivers "find" the optimal throttle position, and thus the wheel torque, to maximize longitudinal traction performance. This task was intentionally simplified for a number of reasons. First, numerical simulation results were easily compared with the

\begin{tabular}{|c|c|c|c|c|}
\hline & \multicolumn{2}{|c|}{ Displacement } & \\
\hline DOF & Single DOF & Maximum & Velocity & Acceleration \\
\hline Surge & $-0.46 /+0.57 \mathrm{~m}$ & $\pm 0.57 \mathrm{~m}$ & $\pm 0.7 \mathrm{~m} / \mathrm{s}$ & $\pm 7 \mathrm{~m} / \mathrm{s}^{2}$ \\
\hline Sway & $\pm 0.47 \mathrm{~m}$ & $\pm 0.5 \mathrm{~m}$ & $\pm 0.7 \mathrm{~m} / \mathrm{s}$ & $\pm 7 \mathrm{~m} / \mathrm{s}^{2}$ \\
\hline Heave & $\pm 0.39 \mathrm{~m}$ & $\pm 0.39 \mathrm{~m}$ & $\pm 0.5 \mathrm{~m} / \mathrm{s}$ & $\pm 10 \mathrm{~m} / \mathrm{s}^{2}$ \\
\hline Roll & $\pm 23.2^{\circ}$ & $\pm 23.8^{\circ}$ & $\pm 34 \% / \mathrm{s}$ & $>225^{\circ} / \mathrm{s}^{2}$ \\
\hline Pitch & $-23.2^{\circ} /+25.5^{\circ}$ & $-27.4^{\circ} /+31.6^{\circ}$ & $\pm 35 \% / \mathrm{s}$ & $>225^{\circ} / \mathrm{s}^{2}$ \\
\hline Yaw & $\pm 24.3^{\circ}$ & $\pm 27.6^{\circ}$ & $\pm 35 \% / \mathrm{s}$ & $>225^{\circ} / \mathrm{s}^{2}$ \\
\hline
\end{tabular}

Table 1. Performance Specifications for the VIPER HIL Driving Simulator

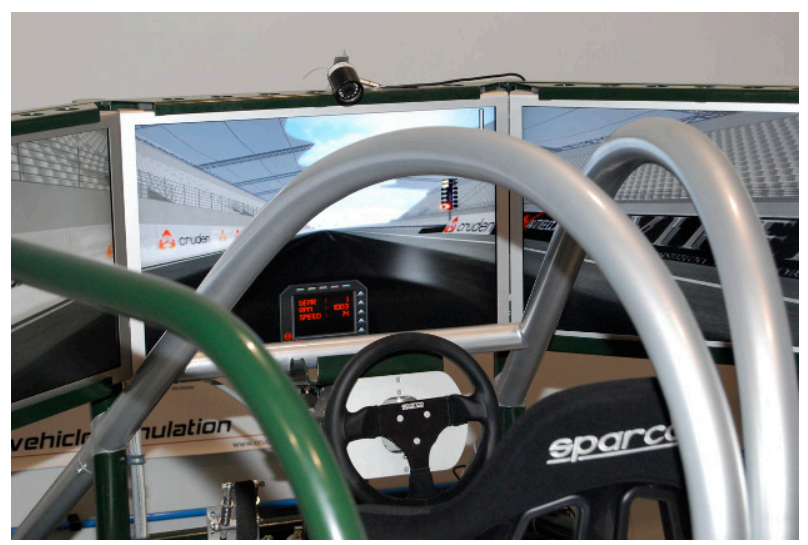

Figure 2. View of the virtual track used for HIL testing on the driving simulator.

experimental HIL test results from a driving simulator, and second, minimizing the number of input and output variables was expected to lead to a more concise understanding of how human experts operate at the uncertain limits of performance. Even with the restrictions associated with this simple driving task, the non-linear traction dynamics at the tireground interface were more than sufficient to make this a challenging yet highly instructive case study.

For this HIL case study, formal test protocols were developed instructing the human expert to drive a particular ground vehicle along a straight line on a flat dry paved surface starting from a resting condition. In addition to minimizing the steering input, the driver was also instructed not to switch gears and not to apply the brakes during any test run. These additional degrees of freedom are quite interesting and will ultimately play a key role when considering both longitudinal and lateral traction control; however, their inclusion would have compromised the ability to isolate desired human behavior in this case study. Mathematically, if we define $T_{25}$ to be the time it takes the simulated vehicle to reach a distance of twenty-five meters, then the formal objective was to control only the throttle input, or wheel torque, to minimize $T_{25}$. Since the driver was intentionally not provided with direct feedback on the $T_{25}$ time, they were required to rely on internal motivation, or to simply "drive as fast as possible for each run."

In order to make this HIL case study more challenging and realistic, a virtual car was selected with enough wheel torque to quickly reach a tire burnout slip condition at any point within the 25 meter test track. The virtual car model parameters and the track surface friction prop- 
erties were chosen to provide the human driver with a realistic driving experience. During the test, the driver experienced visual cues, audio platform motion, and steering force feedback, although little or no steering was actually required for this study. The photo in Figure 2 shows an over-the-shoulder view of the simulated track as seen by a driver on the simulator.

\section{Simulation Model Development}

The simple driving task designed for this case study is commonly studied in the literature using a single-wheel ground vehicle model, which is essentially a quarter-car sans suspension, as indicated in Figure 3. Similar models have been utilized for studying the non-linear longitudinal dynamics of traction control $[14,15]$, as well as alternative traction control algorithms [1,6,16-18].
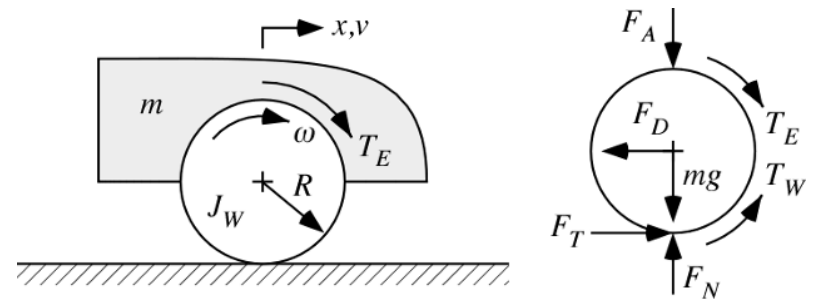

Figure 3. Dynamic model used for simple ground vehicle traction control study

The single-wheel model of Figure 3 is clearly not representative of an actual car; however, it does illustrate the pertinent non-linear longitudinal dynamics associated with wheeled vehicle traction, and more importantly, it models the dynamics of the virtual vehicle on the driving simulator with reasonable accuracy. From Figure 3 , the vehicle mass $m$ is constrained to move longitudinally with displacement $x$ and velocity $v$. This mass is "driven" by applying a controlled torque $T_{E}$ to the single wheel with rotational inertia $J_{W}$, effective radius $R$, and angular velocity $\omega$. The wheel is assumed to be damped with a viscous torque $T_{W}=\beta \omega$. The net horizontal force is the sum of the non-linear traction force $F_{T}$ and an assumed linear aerodynamic drag force: $F_{D}=\alpha v$. Combining these results, the complete linear dynamics are given by equation (1)

$$
\begin{aligned}
{\left[\begin{array}{c}
\dot{v} \\
\dot{\omega} \\
\dot{T}_{E}
\end{array}\right]=} & {\left[\begin{array}{ccc}
-\left(\frac{\alpha}{m}\right) & 0 & 0 \\
0 & -\left(\frac{\beta}{J_{W}}\right) & \left(\frac{1}{J_{W}}\right) \\
0 & 0 & -\left(2 \pi f_{E}\right)
\end{array}\right]\left[\begin{array}{c}
v \\
\omega \\
T_{E}
\end{array}\right]+} \\
& +\left[\begin{array}{cc}
0 & \left(\frac{1}{m}\right) \\
0 & -\left(\frac{R}{J_{W}}\right) \\
\left(2 \pi f_{E}\right) & 0
\end{array}\right]\left[\begin{array}{c}
T_{C} \\
F_{T}
\end{array}\right]
\end{aligned}
$$

where $T_{C}$ is the commanded input torque and $T_{E}$ is the actual applied wheel torque. In this model, the actual applied torque state is assumed to be the output of a first-order unity-gain low-pass filter with break frequency $f_{E}(\mathrm{~Hz})$
Note that the dynamics for this simple single-wheel model are linear except for the constitutive equation for the traction force. The non-linear traction force, $F_{T}$, is given by the semi-empirical "Magic Formula" of Pacejka:

$$
\begin{aligned}
F_{T}(\kappa)= & D\left(F_{N}\right) \sin \left\{C ( F _ { N } ) \operatorname { a r c t a n } \left(\kappa B\left(F_{N}\right)\left[1-E\left(F_{N}\right)\right]+\right.\right. \\
& \left.\left.+E\left(F_{N}\right) \arctan \left[\kappa B\left(F_{N}\right)\right]\right)\right\}
\end{aligned}
$$

where $B, C, D$, and $E$ are respectively the stiffness, shape, peak, and curvature coefficients for a given tire-ground interface $[19,20]$. As indicated in equation (2), these coefficients are dependent on the normal force $F_{N}$, which for most ground vehicles is also a time-varying quantity [20]. Reacting at the tire-ground interface, the normal force $F_{N}$ is the sum of a gravitational force and an aerodynamic down-force, which, like the vehicle model on the simulator is assumed here to be proportional to vehicle speed

$$
F_{N}=m g+F_{A}=m g+\gamma v
$$

The traction force in equation (2) is also dependent on a nondimensional slip ratio $(\boldsymbol{k})$ defined by SAEJ270 $[9,19,20]$ in terms of the velocity states from the linear dynamics

$$
\kappa \equiv\left(\frac{\omega R-v}{v}\right)
$$

Note that $\omega R>v \rightarrow \kappa>0$ during acceleration, and $\omega R<v \rightarrow$ $\kappa<0$ when braking $[2,7]$.

A family of example traction curves is shown in Figure 4 for a tire on three different but uniform terrain surfaces with a constant normal loading force. This plot shows the complete longitudinal traction curves for both acceleration and braking; although, only acceleration is considered in this study. The dashed lines in the curves of Figure 4 represent the regions of wheel slip where the traction force remains high, but not at the peak value. In these slip regions, a greater percentage of the contact patch is slipping as the tire either approaches burnout $(\boldsymbol{k}=1)$ or lockup $(\kappa=-1)$ [9]. It is clear from Figure 4 that minimizing the $T_{25}$ time will require the driver to operate at the peak traction force for as long as possible. In addition to not having any direct feedback such as the slip ratio or the actual traction force, the human driver is faced with an additional challenge because the peak traction force and the slip ratio at which the peak occurs is dynamically changing with normal load as indicated by equation (3). A real tire-ground interface is even more complex since the terrain surface is generally not spatially uniform as was assumed in this case study.

For comparison purposes, Figure 5 shows an example traction control system based on a conventional feedback strategy to directly regulate the slip ratio. The MoTeC M880 Engine Control Unit is an example control system that incorporates a PID-based traction control loop as indicated in Figure 5, and is used in many motorsports applications [29]. Indeed, variations of this approach are used in a variety of commercial automobile traction control systems (TCS) $[1,2,16-18,21,22]$, anti-slip regulation systems (ASR), and anti-lock braking systems (ABS) $[5-7,23]$.

The ground vehicle dynamics in Figure 5 are represented by the linear dynamics of equation (1) coupled with the non-linear traction force 


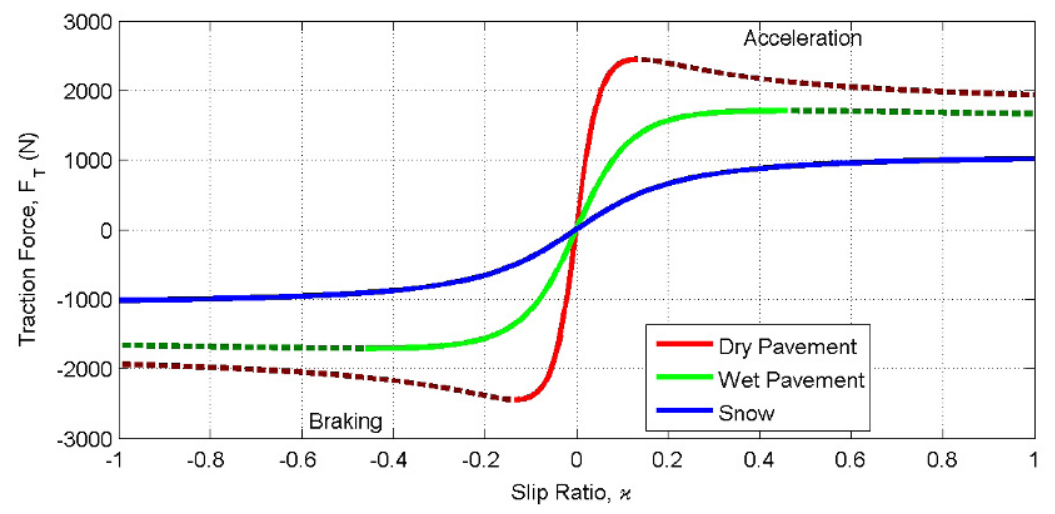

Figure 4. Example traction curves from the Pacejka Magic Formula [7, 20].

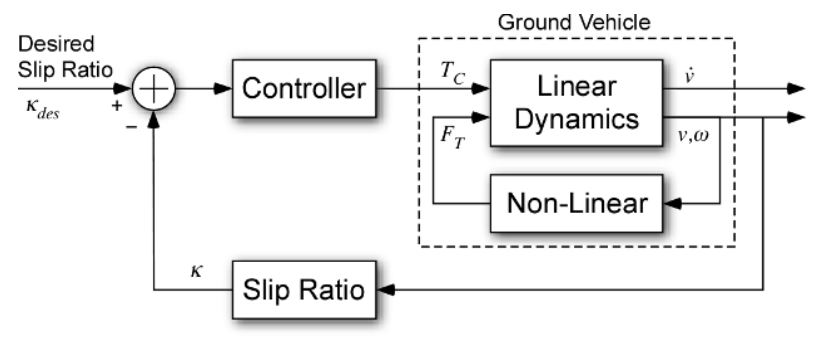

Figure 5. Simulation model with conventional feedback loop to regulate slip.

given by equations (2) - (4). Although a human driver does not sense or have any direct perception of slip ratio, the two velocity states can be measured with physical sensors and then used to compute the slip ratio according to equation (4). Using the Dry Pavement example in Figure 4 , the peak traction force at the nominal normal force occurs near $\kappa=0.133$, so regulating the actual slip ratio to this desired value will essentially maximize the traction force, resulting in a minimized $T_{25}$ time. Unfortunately, this approach does not work well in the very common situation where the terrain surface is not uniform since it is difficult if not impossible to specify an appropriate desired slip ratio to cover the wide range of surface possibilities. Most commercial TCS and ABS systems do not attempt to achieve the peak traction force, typically regulating the slip ratio to sub-optimal levels in order to insure robustness over a wider range of terrain surfaces. For high performance motorsports applications, the MoTeC M880 ECU accepts a potentiometer input which allows the driver to make real-time adjustments to the slip ratio setpoint [29].

\section{Comparison of HIL Testing with PID Traction Control Simulation}

In order to evaluate the results from the human-in-the-loop test on the driving simulator, a PID-based traction control simulation was set up as a benchmark. The PID controller was tuned to provide a closed-loop response with a bandwidth that approximately matched the nominal bandwidth of a human driver actuating the throttle on the driving simulator. Table 2 lists the parameter values used for this simulation study except for the Pacejka coefficients. These parameters are equivalent to the actual parameters associated with the virtual vehicle used during the HIL testing on the driving simulator.

\begin{tabular}{|c|c|c|c|}
\hline Parameter & Value & Units & Description \\
\hline \hline$m$ & 540.0 & $\mathrm{~kg}$ & Mass of virtual vehicle \\
\hline$J_{W}$ & 1.0 & $\mathrm{~kg}-\mathrm{m}^{2}$ & Rotational inertia of driven wheel \\
\hline$R$ & 0.31 & $\mathrm{~m}$ & Effective radius of driven wheel \\
\hline$\alpha$ & 25.0 & $\mathrm{Ns} / \mathrm{m}^{2}$ & Aerodynamic drag constant \\
\hline$\beta$ & 1.0 & $\mathrm{Nms} / \mathrm{rad}$ & Viscous friction in driven wheel \\
\hline$\gamma$ & 60.0 & $\mathrm{~N}-\mathrm{s} / \mathrm{m}^{2}$ & Aerodynamic down-force constant \\
\hline$f_{E}$ & 10.0 & $\mathrm{~Hz}$ & Break frequency of wheel torque low-pass \\
\hline$K_{P}$ & 800.0 & - & Proportional gain for PID \\
\hline$K_{l}$ & 40000.0 & - & Integral gain for PID \\
\hline$\kappa_{\text {des }}$ & 0.133 & - & Desired slip ratio setpoint for PID \\
\hline$\mu$ & 750.0 & - & Adaptation step size for gradient descent \\
\hline$\eta$ & 0.0013 & - & Gradient value for negative gradients \\
\hline
\end{tabular}

Table 2. Parameter values used in the simulation study

During the HIL testing, the human driver attempted fifty-one consecutive runs within the same test period without any "warm-up" trials. All driver inputs and vehicle dynamic responses were recorded during the experimental HIL testing. False starts and otherwise corrupted runs were removed from the data during post-processing, leaving a total of forty-seven usable runs. The four time series plots in Figure 6 summarize the key signals for each of the usable runs. In each plot below, the "best" run, i.e. minimum $T_{25}$ time, is highlighted in green and the "worst" run is highlighted in red. The remaining forty-five runs were sorted by $T_{25}$, and are shaded from light gray (second best) to dark gray (second worst). The response data from the PID simulation model of Figure 5 are plotted as magenta curves in Figure 6 for direct comparison with the experimental HIL responses. 
Figure 6(a) is a plot of the displacement $x$ of the vehicle. Note that reference grid lines have been drawn on this plot as displacement trajectories associated with constant accelerations. For the HIL testing, the best $T_{25}$ time was 2.39 seconds and the worst $T_{25}$ time was 2.99 seconds. Figure $6(\mathrm{~b})$ is a plot of the control input torque $T_{E}$, and Figure $6(c)$ is the corresponding slip ratio, $\boldsymbol{k}$. These simulation results confirm that the best $T_{25}$ solution requires the torque to quickly reach a level where the slip ratio maximizes the traction force. This comparison also confirms that the best $T_{25}$ time for the human driver is not as good as the $T_{25}$ time for the PID simulation because the human driver did not maintain the peak traction force for the same amount of time as the PID simulation. Several interesting observations regarding the behavioral response of the human driver can be gleaned from Figure 6(c). In order to "learn" where the peak tractive force is, the driver must occasionally exceed that limit. In every case where the limit is exceeded, the HIL data indicates that the driver is not only aware of this condition, but also compensates by reducing the throttle. The runs where the driver successfully regulates the throttle such that the traction force is maintained near the peak level have consistently better $T_{25}$ times.

Even with this limited set of runs, the human driver was able to demonstrate an ability to learn. This is depicted more clearly in Figure 7 where the $T_{25}$ times are plotted against sequential run number. The shaded region in Figure 7 is an admittedly subjective estimate of an envelope centered around the data, showing a generally downward trend. The best $T_{25}$ time occurred during run 36, and after making significant progress up to run 30 , the human driver consciously began a series of even more aggressive trials (runs $32,34,39,46$, and 47) which were each immediately followed by one or more good runs. These HIL test results raise a fundamental question: How does the human driver learn to improve his or her performance without direct knowledge of the traction force or wheel slip? The human driver receives feedback from multiple sources such as visual, audible, tactile, and motion. For this simple task, the visual and tactile feedback is very likely negligible by design; however, the audible feedback of tire squeal as well as the longitudinal acceleration of the platform are well correlated to the traction force being generated. From the HIL acceleration data in Figure $6(d)$, it is clear that longitudinal acceleration is reduced whenever the wheel approaches a burnout condition. To confirm this relationship numerically, cross-correlations were computed from the HIL test data between key driver inputs and acceleration response outputs. The normalized cross-correlation between any two sampled-data signals was calculated as

$$
\rho(n) \equiv \frac{\sum_{k} \operatorname{signal}_{1}(k) \operatorname{signal}_{2}(k-n)}{\sqrt{\sum_{k} \operatorname{signal}_{1}^{2}(k)} \sqrt{\sum_{k} \operatorname{signal}_{2}^{2}(k)}},
$$

where $k$ is the sample index and $n$ is the delay or lag between signals. Table shows the results of a zero-lag $(n=0)$ correlation study between the driver input (throttle and resulting wheel torque) and the pertinent acceleration responses sensed by the driver, with the exception of visual and audible feedback. The data in Table 3 shows the mean correlation across all 47 runs, as well as the associated standard deviation.

In the HIL simulation, the throttle is the primary input from the driver; however, Table 3 confirms that the throttle and wheel torque are highly correlated. As expected from inspection of Figure 6 (b) and 6 (d), and as clearly demonstrated in Table 3, the longitudinal acceleration (i.e. Surge Accel) has a significantly higher level of correlation to both the throttle and the wheel torque, than any of the other five accelerations sensed by the driver. Note that the relatively high negative correlation in Table

\begin{tabular}{|ccccr|}
\hline & \multicolumn{2}{c}{ Throttle } & \multicolumn{2}{c|}{ Wheel Torque } \\
\hline & Mean & STD & Mean & STD \\
\hline \hline Throttle & 1.000 & 0.0002 & 0.989 & 0.0041 \\
\hline Wheel Torque & 0.989 & 0.0041 & 1.000 & 0.0004 \\
\hline Surge Accel & 0.981 & 0.0157 & 0.981 & 0.0235 \\
\hline Sway Accel & 0.218 & 0.4145 & 0.236 & 0.4441 \\
\hline Heave Accel & -0.803 & 0.2170 & -0.806 & 0.2209 \\
\hline Pitch Accel & 0.088 & 0.2370 & 0.085 & 0.2433 \\
\hline Roll Accel & -0.138 & 0.2204 & -0.149 & 0.2341 \\
\hline Yaw Accel & 0.145 & 0.4031 & 0.130 & 0.3846 \\
\hline
\end{tabular}

Table 3. Correlation between driver input and acceleration responses.

3 between either the throttle or the wheel torque and the heave acceleration is physically due to the linear relationship between aerodynamic downforce and vehicle speed.

\section{Adaptive Traction Control Algorithm}

HIL test results provided both qualitative and quantitative evidence that the human driver relies on directly sensed longitudinal acceleration as the key feedback to determine the optimal time-varying throttle position for the simple driving task defined above. The HIL experimental test results also indicate that the driver occasionally commands the throttle to exceed the peak traction force. One plausible explanation for this is that the driver is attempting to construct a perceptive model of the relationship between the input (throttle or wheel torque) and the output (longitudinal acceleration).

Our primary objective is to develop a traction control method that commands throttle position (wheel torque) based only on sensed longitudinal acceleration. Since it is also an objective to implement the method on a digital controller, we will first discretize the dynamic vehicle model while retaining the non-linearities. The block diagram in Figure 5 indicates that the linear dynamic system has two outputs and two inputs. This particular structure, i.e. continuous LTI dynamics coupled with a non-linear feedback path, is a generalized non-linear dynamic system representation

$$
\begin{aligned}
& \dot{x}=A_{c} x+B_{c} u+F_{c} u^{\prime} \\
& y=C_{c} x+D_{c} u+G_{c} u^{\prime}
\end{aligned}
$$

and

$$
y^{\prime}=\left[\begin{array}{l}
l \\
0
\end{array}\right] x+\left[\begin{array}{l}
0 \\
l
\end{array}\right] u=\left[\begin{array}{l}
x \\
u
\end{array}\right]
$$

where the subscript-c identifies the matrices as continuous-time elements, $y \in \Re^{P}$ represents a vector of sensed outputs, and the internal output vector $y^{\prime} \in \Re^{N+M}$ contains the state vector $x \in \Re^{N}$ augmented with the input vector $u \in \Re^{M}$. The non-linear internal feedback input $u^{\prime} \in \Re^{Q}$ can always be chosen such that

$$
u^{\prime}=f\left(y^{\prime}\right)=\left[\begin{array}{lll}
f_{1}\left(y^{\prime}\right) & \cdots & f_{Q}\left(y^{\prime}\right)
\end{array}\right]^{T}
$$



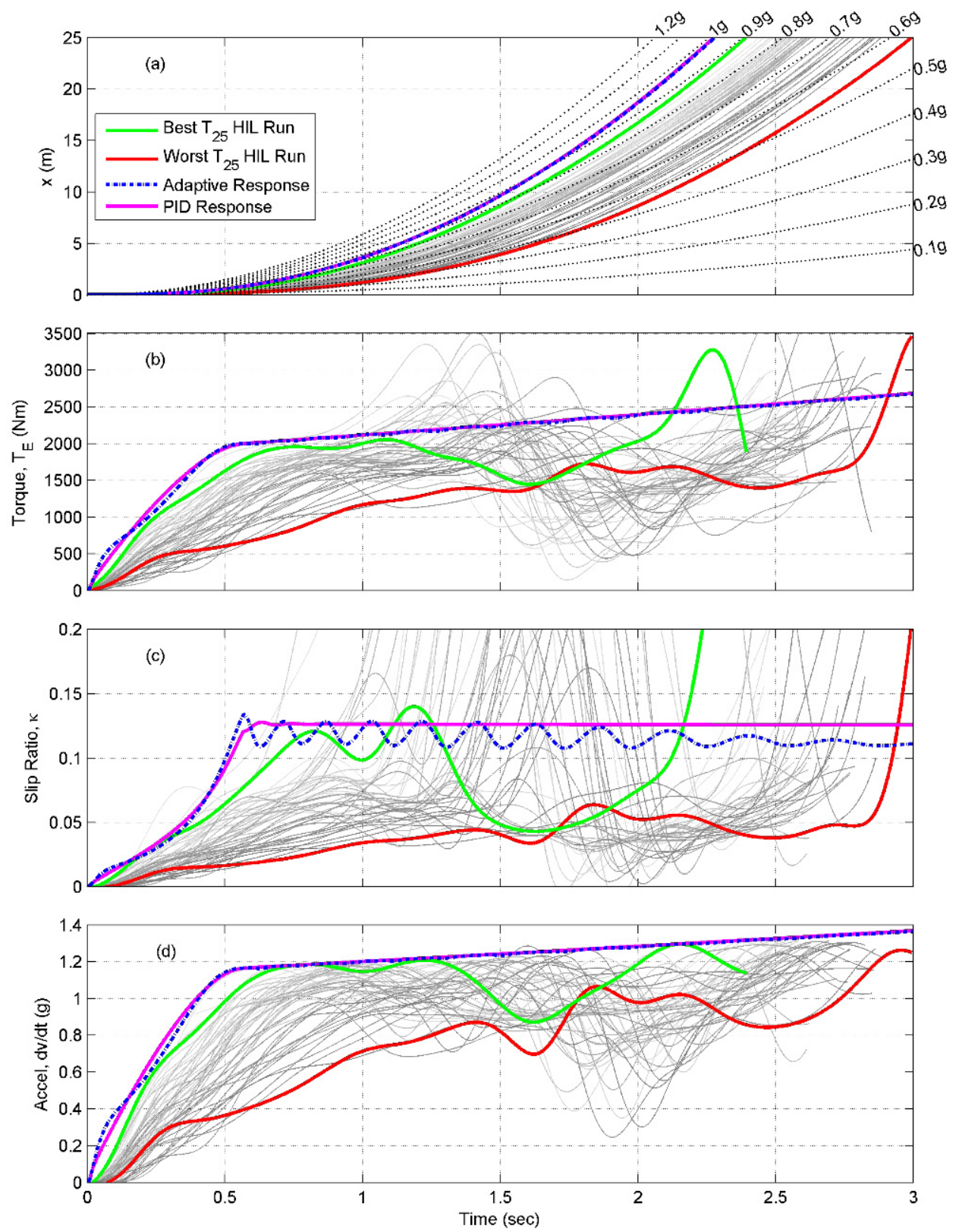

Figure 6. HIL experimental data compared to PID and adaptive simulation results.

where each $f_{q}\left(y^{\prime}\right)$ is a general memoryless non-linear scalar function of the states and inputs. Within the general framework of equations (6) and (7), the simulation model for this particular case study results in the following specific definitions

$u=\left[T_{C}\right] \in \Re^{1}$

$u^{\prime}=\left[F_{T}(v, \omega)\right] \in \Re^{1}$

$y=[\dot{v}] \in \Re^{1}$

$F_{c}=\left[\begin{array}{lll}\frac{1}{m} & -\frac{R}{J_{W}} & 0\end{array}\right]$

$G_{c}=\left[\frac{1}{m}\right]$ 


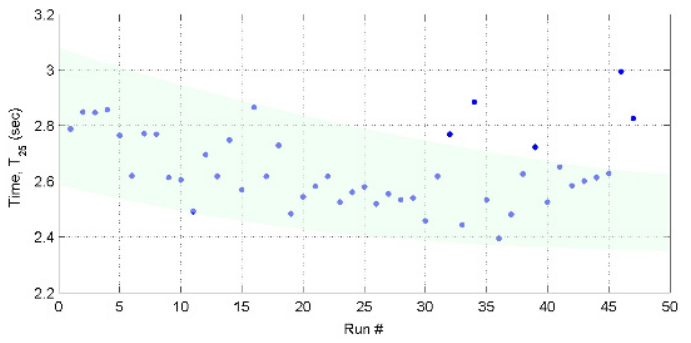

Figure 7. Learning trend from HIL consecutive run testing.

The control algorithm development will proceed using the general formulation in order to enable a wider scope of applicability than the simple task in this case study.

The LTI dynamics in equation (6) can be discretized using any of a number of methods such as the Tustin bilinear transform. The resulting discrete time equation incorporating the non-linear feedback is given by

$$
\begin{gathered}
x_{k+1}=A x_{k}+B u_{k}+F u_{k}^{\prime}, \\
y_{k}=C x_{k}+D u_{k}+G u_{k}^{\prime}, \\
u_{k}^{\prime}=f\left(y_{k}^{\prime}\right),
\end{gathered}
$$

where the non-linear feedback term (9b) is unaffected by the discretization process since it contains no dynamic elements. The discrete-time formulation of equation (9a) will facilitate simulation of the complete non-linear system without linearization error, and it will also enable a convenient means for developing the control law.

Based on observations from the HIL test data and the correlation study (Table 3), a gradient-based approach is proposed as a candidate solution for determining the optimal throttle position to maximize longitudinal acceleration. There are many possible gradient-based approaches that can be employed; however, for this study, a steepest ascent approach was chosen. The basic discrete-time control algorithm structure has the following form

$$
u_{k+1}=u_{k}+\mu \nabla_{k},
$$

where $\mu$ is a constant step-size that controls the rate of convergence, and $\nabla_{k}$ is the gradient of a positive definite quadratic objective function of the outputs. For this case study the only sensed output used for feedback is the longitudinal acceleration, therefore

$$
J\left(u_{k}\right)=\frac{1}{2}\left(\dot{v}_{k}\right)^{2}=\frac{1}{2} y_{k}^{T} y_{k}
$$

From equation (9a), it is clear that the objective function defined by equation (11) is dynamically dependent on the control input, $u_{k}$. The objective of traction control is thus to find a command $u_{k}$ that maximizes this objective function. Using the objective function definition from equation (11), the gradient is evaluated as

$$
\nabla_{k}=\frac{\partial J\left(u_{k}\right)}{\partial u_{k}}=\left(\frac{\partial y_{k}}{\partial u_{k}}\right)^{T} y_{k}
$$

Since $y_{k}$ is a measured quantity, it is readily available for input to the adaptation algorithm; however, the gradient term $\left(\partial y_{k} / \partial u_{k}\right)$ requires evaluation of the system dynamics given by equation (9a), including the non-linear feedback terms (9b). As will be shown below, this gradient term represents a dynamic "filtering" operation as is commonly found in Filtered-X LMS algorithm implementations [24].

In order to evaluate the dynamic partial derivative term in equation (12), we first define the following LTI $z$-transform matrix operator: $H(z) \equiv[z I-A]^{-1}$. Using this operator, the LTI difference-equation from equation (9a) can be transformed, resulting in

$$
\left[\begin{array}{l}
x(z) \\
y(z)
\end{array}\right]=\left[\begin{array}{cc}
H(z) B & H(z) F \\
C H(z) B+D & C H(z) F+G
\end{array}\right]\left[\begin{array}{c}
u(z) \\
u^{\prime}(z)
\end{array}\right] .
$$

Evaluating the partial derivative term in equation (12) using the transformed expression for the output vector $y$ from equation (13) yields the following

$$
\left(\frac{\partial y(z)}{\partial u(z)}\right)=[C H(z) B+D]+[C H(z) F+G]\left(\frac{\partial u^{\prime}(z)}{\partial u(z)}\right)
$$

From the definition of the non-linear feedback term in equation (9b), the partial derivative term on the RHS of equation (14) can be expanded using the chain rule

$$
\begin{array}{r}
\left(\frac{\partial u^{\prime}(z)}{\partial u(z)}\right)=\left(\frac{\partial f(x(z), u(z))}{\partial u(z)}\right)= \\
=\left(\frac{\partial f(x, u)}{\partial u(z)}\right)+\left(\frac{\partial f(x, u)}{\partial x(z)}\right)\left(\frac{\partial x(z)}{\partial u(z)}\right) .
\end{array}
$$

The partial derivative of the state vector with respect to the control input vector in equation (15) can also be expanded using the state equation result from (13)

$$
\left(\frac{\partial x(z)}{\partial u(z)}\right)=H(z) B+H(z) F\left(\frac{\partial u^{\prime}(z)}{\partial u(z)}\right) .
$$

Notice that equations (15) and (16) are recursively coupled. This algebraic loop can be broken by substituting equation (15) into (16) and rearranging terms to solve for

$$
\begin{aligned}
\left(\frac{\partial x(z)}{\partial u(z)}\right)= & {\left[I-H(z) F\left(\frac{\partial f(x, u)}{\partial x(z)}\right)\right]^{-1} } \\
& H(z)\left[B+F\left(\frac{\partial f(x, u)}{\partial u(z)}\right)\right] .
\end{aligned}
$$

The general expression for the dynamic gradient term in equation (12) is finally obtained by substituting equations (15) and (17) into equation (14), resulting in

$$
\begin{aligned}
\frac{\partial y}{\partial u}= & {[C H B+D]+[C H F+G]\left[\left(\frac{\partial f}{\partial x}\right)[I-\right.} \\
& \left.\left.-H F\left(\frac{\partial f}{\partial x}\right)\right]^{-1} H\left[B+F\left(\frac{\partial f}{\partial u}\right)\right]+\left(\frac{\partial f}{\partial u}\right)\right],
\end{aligned}
$$


where the dependence on $z$ has been omitted for brevity. Most of the terms in equation (18) are dynamic LTI matrix operators. In order to implement this result in the real-time update law of equation (10), the individual dynamic terms in equation (18) must be represented in the time-domain. The time-domain implementation is actually much simpler than equation (18) might suggest.

The terms in equation (18) involving partial derivatives of the nonlinear function (9b) with respect to states and inputs, represent quasiconstant transformation matrices. To illustrate this fact using the quarter-car model defined by equations (1) - (4), these partial derivatives are simply given by

$$
\begin{aligned}
& \frac{\partial f\left(x_{k}, u_{k}\right)}{\partial x}=\left[\begin{array}{lll}
\frac{\partial F_{T}\left(v_{k}, \omega_{k}\right)}{\partial v} & \frac{\partial F_{T}\left(v_{k}, \omega_{k}\right)}{\partial \omega} & \frac{\partial F_{T}\left(v_{k}, \omega_{k}\right)}{\partial T_{C}}
\end{array}\right] \\
& \text { and } \\
& \frac{\partial f\left(x_{k}, u_{k}\right)}{\partial u}=0
\end{aligned}
$$

Assuming that the parameter values for the Pacejka model of equation (2) are known, the derivatives in equation (19) are easily evaluated at the current state; however, they must be re-evaluated at each time step.

The remaining terms in equation (18) are MIMO dynamic systems. The first two terms in (18) are easily recognized as linear time-invariant state-space representations

$$
r^{(1)}(z)=[C H(z) B+D] q^{(1)}(z) \Rightarrow \begin{gathered}
x_{k+1}^{(1)}=A x_{k}^{(1)}+B q_{k}^{(1)} \\
r_{k}^{(1)}=C x_{k}^{(1)}+D q_{k}^{(1)}
\end{gathered},
$$

$$
r^{(2)}(z)=[C H(z) F+G] q^{(2)}(z) \Rightarrow \begin{gathered}
x_{k+1}^{(2)}=A x_{k}^{(2)}+F q_{k}^{(2)} \\
r_{k}^{(2)}=C x_{k}^{(2)}+G q_{k}^{(2)}
\end{gathered},
$$

where the $x_{k}^{(i)}$ are internal state vectors, $q_{k}^{(i)}$ are input vectors, and $r_{k}^{(i)}$ are output vectors of the respective dynamic systems. The final bracketed dynamic term in equation (18) requires more algebraic manipulation, but is still easily represented by a linear slowly time-varying statespace system.

$$
\begin{gathered}
x^{(3)}(z)=\left[\left(\frac{\partial f}{\partial x}\right)\left[I-H F\left(\frac{\partial f}{\partial x}\right)\right]^{-1} H\left[B+F\left(\frac{\partial f}{\partial u}\right)\right]\right] q^{(3)}(z) \\
\Rightarrow \begin{array}{l}
x_{k+1}^{(3)}=\left[A+F\left(\frac{\partial f}{\partial x}\right)\right] x_{k}^{(3)}+\left[B+F\left(\frac{\partial f}{\partial u}\right)\right] q_{k}^{(3)} \\
r_{k}^{(3)}=\left(\frac{\partial f}{\partial x}\right) x_{k}^{(3)}+\left(\frac{\partial f}{\partial u}\right) q_{k}^{(3)}
\end{array}
\end{gathered}
$$

To evaluate the adaptation law given by equation (10), the gradient defined in equation (12) is computed at each time step by "filtering" the input vector $y_{k}$ through the series and parallel combination of dynamic systems defined by equations (18), (20), and (21).

\section{Adaptive Traction Control Simulation Comparison}

The adaptive traction control law derived in the previous section was implemented for the case study example in a second simulation for direct comparison with the HIL data and the benchmark PID simulation data presented above. All simulations were performed with the same set of parameters given in Table 2, and also the same Pacejka tire model for Dry Pavement (Figure 4) that was used in the HIL testing on the driving simulator.

Initial evaluation of the adaptive traction control simulation results indicated the need for a minor adjustment to the update law. The modified control law is given by

$$
u_{k+1}=u_{k}+\mu \times\left\{\begin{array}{cc}
\nabla_{k} & \nabla_{k}>0 \\
-\eta & \nabla_{k} \leq 0
\end{array},\right.
$$

where $\eta>0$ is a constant value (Table 2) which is used to represent the gradient value whenever the gradient is negative. With regard to the case study example, a negative gradient physically corresponds to the situation where the slip ratio is beyond the peak point in the traction curve and the update law is effectively attempting to reduce the throttle or wheel torque. Notice from equation (22) that when the gradient is positive, the update law is identical to the original control law in equation (10). This modification is known in the adaptive signal processing field as a "Signed Regressor" variant [25]. The justification for this modification can be explained with reference to Figure 4. Specifically, for the Dry Pavement traction curve at relatively high slip ratios (i.e. dashed line region), the gradient is negative but small and even approaching zero. In order to speed up convergence in this region to push the slip ratio back toward the peak, the magnitude of the actual gradient is replaced by an appropriately chosen constant.

Simulation results for the proposed adaptive traction control law are plotted as blue dashed lines in Figure 6 for direct comparison with the PID control solution and the HIL data. Figures $6(a), 6(b)$, and $6(d)$ indicate that the PID controller and the adaptive controller both produce similar results, which are very nearly optimal. From the Pacejka tire model of equation (2), the tractive force is a function of normal load, which effectively means that the Dry Pavement traction curve in Figure 4 will vary with changes in the normal load. For all three simulations in this case study, equation (3) indicates that the aerodynamic down force, and therefore the normal load, increases linearly with velocity. Pacejka [19, 20] and Bakker [26] have shown that as the normal load increases, the slip ratio associated with the peak tractive force will decrease in magnitude. This phenomena is clearly seen in Figure 6(c) where the adaptive solution automatically tracks this change in optimum slip ratio $k$, whereas the PID control solution is not designed to do so and therefore must be de-tuned to insure robust performance. The adaptive traction control response shown in Figure 6(c) also indicates what appears to be a hunting behavior around the optimal slip ratio. The magnitude of the hunting oscillation can be increased or decreased with the step size selection; however, this hunting behavior is a key advantage of the proposed algorithm. Although this response is similar to the desirable behavioral response of a human, it was not intentionally designed into the algorithm. The fact that it is occurring can be attributed to the well-known misadjustment behavior of fixed step size gradient-based adaptive algorithms [27]. The ability to continually hunt for an optimal solution is highly desirable since it enables the algorithm to automatically and rapidly adjust to time-varying interface conditions. 

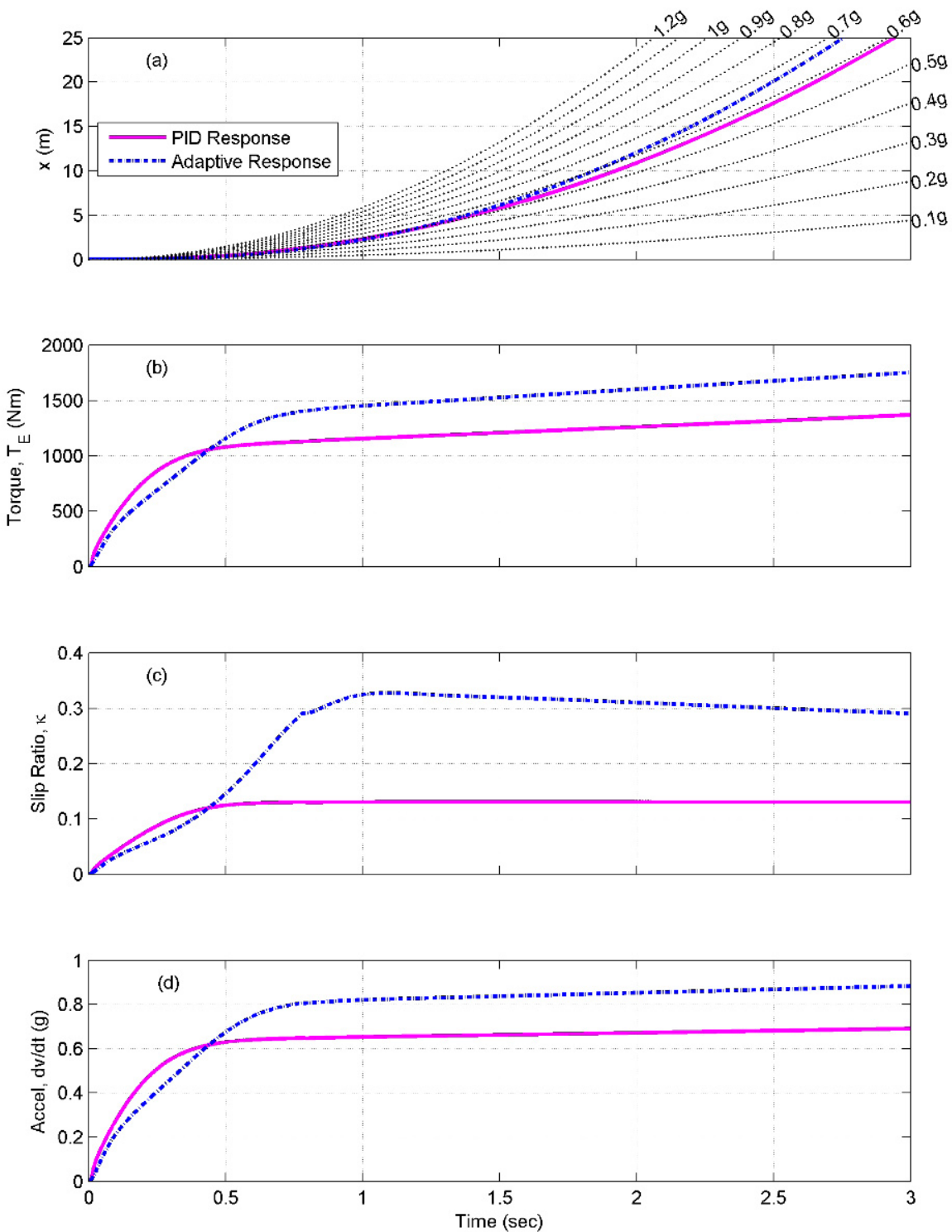

Figure 8. Comparison of the proposed adaptive traction control algorithm with PID on a wet pavement.

The results in Figure 6 indicate that the adaptive traction control algorithm can match the performance of a well-tuned PID loop. To illustrate the advantages of the proposed adaptive algorithm over PID, an additional simulation study was performed using the same two control control algorithms, where the only change was the use of a Pacejka model for wet pavement (Figure 4) to represent the tire-ground inter- face condition. Referring to Figure 4, it is clear that the optimal slip ratio for wet pavement is significantly higher $(0.46)$ than for dry pavement $(0.13)$ with the given normal load condition. It can readily be seen from Figure 8(c) that the adaptive traction control algorithm automatically tracks the slip ratio at the peak traction condition, whereas the $\mathrm{PID}$ solution is only able to regulate to the predetermined set point from 
Table 2. The performance of the proposed adaptive algorithm is superior to the PID algorithm since it allows the vehicle to automatically achieve maximum longitudinal acceleration as indicated in Figure 8(d), which subsequently enables the vehicle to get from point $A$ to $B$ in the minimum amount of time as confirmed by Figure 8(a).

Direct comparison of the results from Figure 6(c) with Figure 8(c) indicates that the dry pavement simulation exhibits a more pronounced hunting behavior than the wet pavement simulation. This result can be attributed to the degree of concavity (i.e. magnitude of the second derivative) in the region of the traction curve in Figure 4 that is near the peak traction force. Dry pavement has significantly more concavity than wet pavement near the respective peak traction levels. The amount of concavity directly affects the magnitude of the gradient in the adaptive algorithm, which translates to increased levels of hunting on dry pavement.

From equations (22), (12), (18), (20), (21), and (22), it is clear that the proposed adaptive traction control algorithm is model-based. Human drivers do not utilize the same types of engineering models such as the model developed above for the proposed traction control algorithm however, it is highly likely that humans do construct perceptive models of not only the vehicle dynamics, but also models of the tire-ground interface. While it is reasonable to expect to have a good understanding of the vehicle dynamics model and associated parameter values, the traction curve associated with the tire-ground interface is less likely to be known to the same level of accuracy, and it will usually be spatially varying as the road conditions change. Kazemi [6], Li [8], Tan [17], and Pasterkamp [28] have developed online identification methods that are specifically designed for estimating the tire traction curve in real-time. These algorithms could be incorporated with the proposed adaptive traction control algorithm to enable an adaptive solution.

\section{Conclusions}

A series of human-in-the-loop tests were performed on a full-motion driving simulator to evaluate and characterize the response of a driver executing a simple high-performance driving task using a virtual but realistic vehicle. Starting from a zero velocity condition, the driver completed a simple repetitive task of driving twenty-five meters with the explicit goal of minimizing the $T_{25}$ time. Based on a correlation analysis of the HIL response data, the human driver was able to demonstrate improved behavior by learning to operate near peak traction levels using longitudinal acceleration as the primary feedback mechanism.

As a first step toward anthropomimetic control system synthesis, a general approach was developed from observations of the HIL response data. The proposed adaptive traction control algorithm was developed with a high level of generality regarding the number and type of feedback sensors, the number and type of control inputs, and non-linearity in the vehicle and tire models. HIL testing confirmed that highly correlated sensors should be used as feedback signals for traction control.

The simulated response of the proposed algorithm was compared against a well-tuned PID benchmark and the HIL response data. Both the proposed adaptive control approach and the PID benchmark require some knowledge of the non-linear traction force; however, for the case study in this paper, the adaptive approach only requires longitudinal acceleration as a feedback signal, just like the human driver utilized. The $T_{25}$ performance comparison indicated that the proposed adaptive algorithm and the PID benchmark were virtually identical on dry pavement; however, the adaptive algorithm was able to maximize traction even when the interface conditions changed to a wet pavement.
Important differences were also observed in the slip ratio responses between the PID and adaptive solutions. The adaptive approach exhibited a hunting behavior around the peak traction condition, which is expected when using a fixed step-size gradient approach. This hunting behavior provides the adaptive algorithm with a significant advantage in that it is always poised to track changes in the interface conditions. The adaptive approach also appeared to indirectly and automatically track the time-varying optimal slip ratio as the increase in vehicle speed produced an increase in aerodynamic downforce resulting in naturally higher traction levels and a shift in the optimal slip ratio. The PID benchmark was not capable of achieving this level of performance.

Even though this research effort did not attempt to explicitly model human behavior, the resulting adaptive control solution exhibited distinct similarities with measured human responses. The performance of an automatic traction control system was clearly improved by incorporating desirable behavioral characteristics. Future research will investigate application of the adaptive traction control algorithm on more complex driving scenarios where braking and steering inputs will be included with the throttle input to maximize both longitudinal and lateral traction.

\section{Acknowledgement}

The authors wish to thank the Institute for Advanced Learning and Research for their support of this research project.

\section{References}

[1] Lee, H. and M. Tomizuka,(2003). "Adaptive vehicle traction force control for intelligent vehicle highway systems (IVHSs)." IEEE Transactions on Industrial Electronics,v. 50: pp. 37-47.

[2] Colli, V., G. Tomassi, and M. Scarano,(2006). "Single Wheel longitudinal traction control for electric vehicles." IEEE Transactions on Power Electronics,v.: pp. 799-808.

[3] Clark, S.K., Mechanics of pneumatic tires. Washington, D.C.: U.S. Dept. of Transportation, National Highway Traffic Safety Administration 1981

[4] Lyon, K., M. Philipp, and E. Grommes,(1994). "Traction Control for a Formula 1 Race Car: Conceptual Design, Algorithm Development, and Calibration Methodology." SAE Motorsports Engineering Conference \& Expostion, December 1994, Dearborn, $\mathrm{MI}$, USA

[5] Instrell, G.K.,(1986). "Anti-Lock Brake Systems - Field Experience on Passenger Cars." Proceedings of the Institution of Mechanical Engineers. Part D, Transport engineering,v. 200: pp. 283-286.

[6] Kazemi , R., B. Hamedi, and B. Javadi,(2000). "A New Sliding Mode Controller for Four-Wheel Anti-Lock Braking System (ABS)." SAE 2000 Automotive Dynamics \& Stability Conference, May 2000, Detroit, MI, USA

[7] Straitiff, D.G., J.S. Freeman, and S.A. Velinsky,(1987)."Simple Methodology for Evaluating and Implementing Vehicle Anti-Lock Brake Systems." Trends in Vehicle Design Research - 1987. Presented at the Winter Annual Meeting of the American Society of Mechanical Engineers.,v. 11: pp. 35-43

[8] Li, K., J.A. Misener, and K. Hedrick,(2007). "On-board road condition monitoring system using slip-based tyre-road friction estimation and wheel speed signal analysis." Proceedings of the Insti- 
tution of Mechanical Engineers, Part K: Journal of Multibody Dynamics,v. 221: pp. 129-146.

[9] Milliken, W.F. and D.L. Milliken, Race Car Vehicle Dynamics. Warrendale, PA: SAE International. 1995

[10] Tran, T.H. and Q.P. Ha,(2008)."Fast algorithm for UGV wheelterrain interaction analysis." 2008 10th International Conference on Control, Automation, Robotics and Vision (ICARCV 2008), 17-20 Dec. 2008 pp. 674-680.

[11] Marques, H.G., R. Newcombe, and O. Holland,(2007). "Controlling an anthropomimetic robot: A preliminary investigation." Lecture Notes in Aritificial Intelligence,v. 4648: pp. 736-745.

[12] Knight, R. and O. Holland, The Anthropomimetic Principle. 2006

[13] Viper Service. Available from:www.viperservice.com.

[14] Olson, B.J., S.W. Shaw, and G. Stepan,(2003). "Nonlinear dynamics of vehicle traction." Vehicle System Dynamics,v. 40: pp. 377-399

[15] Olson, B.J., S.W. Shaw, and G. Stepan,(2005). "Stability and bifurcation of longitudinal vehicle braking." Nonlinear Dynamics,v. 40: pp. 339-65.

[16] Cheok, K., F. Hoogterp, and W. Fales,(1996). "Fuzzy Logic Approach to Traction Control Design." SAE International Congress \& Exposition, February 1996, Detroit, MI, USA.

[17] Tan, H.S. and M. Tomizuka,(1990). "Discrete-time controller design for robust vehicle traction." IEEE Control Systems Magazine,v. 10: pp. 107-13.

[18] Chen, H., J. Yang, Z. Du, and W. Wang,(2010). "Adhesion Control Method Based on Fuzzy Logic Control for Four-Wheel Driven Electric Vehicle." SAE 2010 World Congress \& Exhibition, April 2010, Detroit, MI, USA,V.

[19] Pacejka, H.B. and E. Bakker,(1993). "Magic formula tyre model." Vehicle System Dynamics,v. 21: pp. 1-18.

[20] Pacejka, H.B. and I.J.M. Besselink,(1997). "Magic Formula Tyre Model with Transient Properties "Vehicle System Dynamics International Journal of Vehicle Mechanics and Mobility,v. 27: pp. 234-249.

[21] Wang, H.,(2004). "Hardware-in-the-loop Simulation for Traction Control and the Debugs of its Electric Control Unit." SAE Tech- nical Paper Series,v. 2004-01-2056.

[22] Zhejun, F., K. Yoram, and D. Wehe,(1995)."A simple traction control for tracked vehicles." Proceedings of 1995 American Control Conference - ACC'95, 21-23 June 1995,v. vol.2:pp. 1176-7

[23] Day, T.D. and S.G. Roberts,(2002). "A Simulation Model for Vehicle Braking Systems Fitted with ABS." SAE 2002 World Congress \& Exhibition, March 2002, Detroit, MI, USA,v.

[24] Morgan, D.R. and C. Sanford,(1992). "A control theory approach to the stability and transient analysis of the filtered-x LMS adaptive notch filter." IEEE Transactions on Signal Processing,v. 40: pp. 2341-2346.

[25] Clarkson, P.M., Optimal and Adaptive Signal Processing: CRC Press. 1993

[26] Bakker, E., L. Nyborg, and H.B. Pacejka,(1987). "Tyre modelling for use in vehicle dynamics studies." SAE Technical Paper Series,v. 870421.

[27] Widrow, B. and S. Stearns, Adaptive Signal Processing. Englewood Cliffs, New Jersey: Prentice-Hall. 1985

[28] Pasterkamp, W.R. and H.B. Pacejka,(1997)."Application of neural networks in the estimation of tire/road friction using the tire as sensor." Proceedings of the 1997 International Congress and Exposition, Febrary 24, 1997 - Febrary 27, 1997,v. 1228: pp. $1-7$

[29] MoTeC 880 User Manual, November 2003

[30] Bürki-Cohen, J., Soja, N., and Longridge, T., "Simulator Platform Motion - The Need Revisited," The Int. J. of Aviation Psychology, v 8, n 3, 1998, pp. 293-317

[31] Hoskins, A.H., El-Gindy, M., "Technical Report: Literature Survey on Driving Simulator Validation Studies," Int. J. of Heavy Vehicle Systems, v 13, n 3, 2006, pp. 241-252.

[32] Reymond, G., and Kemeny, A., "Motion Cueing in the Renault Driving Simulator," Vehicle System Dynamics, v 34, 2000, pp. 249-259

[33] Draper, M., Viire, E., Furness, T., and Gawron, V., "Effects of Image Scale and System Time Delay on Simulator Sickness within HeadCoupled Virtual Environments," Human Factors, v. 43, n 1, 2001, pp. $129-146$ 